\title{
Two $\alpha$ Subunit Donor Splice Site Mutations Cause Human Trifunctional Protein Deficiency
}

\author{
Jeffrey C. Brackett, * Harold F. Sims, ${ }^{\ddagger}$ Piero Rinaldo," Scott Shapiro, ${ }^{\star}$ Cynthia K. Powell, ${ }^{\star}$ Michael J. Bennett," \\ and Arnold W. Strauss $\$ \$$ \\ Departments of $*$ Medicine, ${ }^{\ddagger}$ Pediatrics, and ${ }^{8}$ Molecular Biology and Pharmacology, Washington University School of Medicine \\ and St. Louis Children's Hospital, St. Louis, Missouri 63110; "Department of Genetics, Yale University School of Medicine, \\ New Haven, Connecticut 06510; and 'Children's Medical Center, Dallas, Texas 75235
}

\begin{abstract}
Human trifunctional protein catalyzes three steps in mitochondrial $\beta$-oxidation of fatty acids, including the long chain 3-hydroxyacyl-CoA dehydrogenase step. Deficiency of this heterocomplex, which contains $4 \alpha$ and $4 \beta$ subunits, causes sudden unexplained infant death, a Reye-like syndrome, cardiomyopathy, or skeletal myopathy. We determined the molecular basis of this deficiency in a patient with neonatal presentation and later sudden death using reverse transcription and PCR amplification of his $\alpha$ subunit mRNA. We demonstrated a universal deletion of exon 3 (71 bp) in his mRNA. This deletion causes a frameshift and very early premature termination. Amplification of genomic DNA demonstrated that the patient was a compound heterozygote with two different mutations in the $5^{\prime}$ donor splice site following exon 3: a paternally inherited $G$ to $A$ transversion at the invariant position +1 and a maternally inherited $A$ to $G$ mutation at position +3. Both allelic mutations apparently cause exon 3 skipping, resulting in undetectable levels of $\alpha$ subunit protein, and complete loss of trifunctional protein. This is the initial molecular characterization of trifunctional protein deficiency. (J. Clin. Invest. 1995. 95:2076-2082.) Key words: mitochondria - $\beta$-oxidation - sudden death • inborn error $\bullet$ fatty acid metabolism
\end{abstract}

\section{Introduction}

Mitochondrial $\beta$-oxidation of fatty acids provides the primary source of energy in the heart and other high energy-requiring tissues. The $\beta$-oxidation cycle degrades long chain fatty acylCoA substrates via four enzymatic activities to produce acetyl$\mathrm{CoA}$ and a fatty acyl-CoA reduced in length by two carbons. However, because of the wide range of fatty acyl substrate lengths, multiple enzymes with different, but overlapping, substrate specificities catalyze each step. Over the past $15 \mathrm{yr}$, deficiencies in more than 15 human enzymes and proteins essential for mitochondrial fatty acid transport and $\beta$-oxidation have been defined ( 1 ), usually by measurements of accumulated abnormal

Address correspondence to Dr. Arnold W. Strauss, Department of Pediatrics, St. Louis Children's Hospital, One Children's Place, St. Louis, MO 63110. Phone: 314-454-2284; FAX: 314-454-2476.

Received for publication 26 July 1994 and in revised form 28 November 1994.

J. Clin. Invest.

(c) The American Society for Clinical Investigation, Inc. 0021-9738/95/05/2076/07 \$2.00

Volume 95, May 1995, 2076-2082 metabolites and enzymatic assays. The defects are recessively inherited because all of these proteins are encoded by the nuclear genome. Clinical manifestations include a Reye-like syndrome, with hypoglycemia, fatty liver, coma, and frequently death; sudden, unexplained death, usually in the first $2 \mathrm{y}$ of life; dilated or hypertrophic cardiomyopathy; or skeletal myopathy. Among the most common of these known defects is medium chain acyl-CoA dehydrogenase (MCAD) ${ }^{1}$ deficiency, which we and others have delineated at the molecular level $(2,3)$. MCAD is one of four dehydrogenases with overlapping substrate specificities that catalyze the first step of the $\beta$-oxidation spiral.

Deficiency of long chain 3-hydroxyacyl-CoA dehydrogenase (LCHAD) activity in patients with similar presentations was suggested by recent observations of 3-hydroxydicarboxylic aciduria (4-6) and subsequently documented by enzymatic assays $(7,8)$. These biochemical studies suggest that LCHAD deficiency is a relatively common $\beta$-oxidation defect. More recently, deficiency of LCHAD in infants with unexplained sudden death has been associated with the occurrence of maternal acute fatty liver of pregnancy (AFLP) syndrome $(9,10)$.

Elucidation of the molecular basis of LCHAD deficiency required the isolation and characterization of the enzyme (11, 12). Surprisingly, LCHAD activity was discovered as part of a membrane-associated multienzyme complex, designated trifunctional protein. The rat protein is a heterooctamer (12) consisting of $4 \alpha$ and $4 \beta$ subunits. cDNA cloning and expression of rat subunits (13) proved that the $\alpha$ subunit has long chain enoyl-CoA hydratase and LCHAD activities, and the $\beta$ subunit has long chain 3-ketoacyl-CoA thiolase activity.

Enzymatic assays for these three activities in cells from LCHAD-deficient patients demonstrated two types of defects (14). In most individuals, isolated LCHAD deficiency exists with relative preservation of long chain hydratase and thiolase activities. In one such patient, biosynthetic and immunoblotting studies documented normal rates of subunit synthesis with preservation of both subunits (antigen levels $60 \%$ of normal) (14). In other patients, combined deficiency of all three activities is present $(14,15)$, and immunoblot analysis revealed the virtual absence of both $\alpha$ and $\beta$ subunits, presumably because the inability to form a stable complex allows their rapid degradation.

We have recently isolated and characterized the human trifunctional $\alpha$ subunit cDNA and gene. ${ }^{2}$ With these data available,

1. Abbreviations used in this paper: AFLP, acute fatty liver of pregnancy; LCHAD, long chain 3-hydroxyacyl-CoA dehydrogenase; MCAD, medium chain acyl-CoA dehydrogenase; RT-PCR, reverse transcriptase-PCR; SSCV, single-stranded conformation variance.

2. Powell, C. K., H. F. Sims, and A. W. Strauss, unpublished data. 

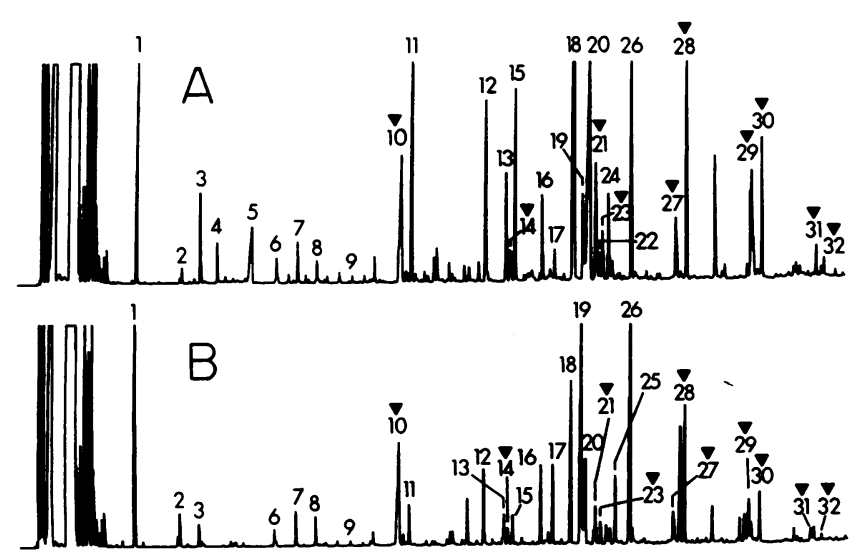

\section{TIME (min)}

Figure 1. Capillary gas chromatographic profiles of urine organic acid trimethylsilyl derivatives in a patient with trifunctional protein deficiency. $(A)$ The profile from a sample obtained at $6 \mathrm{~d}$ of age, during presentation with hypoglycemia and severe myocardial dysfunction (urine volume equivalent to $0.25 \mathrm{mg}$ of creatinine). $(B)$ The profile in a postmortem sample (urine volume equivalent to $1.0 \mathrm{mg}$ of creatinine). Arrowheads mark the positions of peaks containing C6-C14 3-hydroxydicarboxylic acids. With the exceptions of 3-hydroxyadipic lactone (peak 10) and 3-hydroxysebacic acid (peak 28), these metabolites are not detected in normal control specimens. The numbered peaks are as follows: (1) lactic acid; (2) oxalic acid; (3) mixture of 3-hydroxybutyric acid (about $40 \%$ of the peak) and 3-hydroxyisobutyric acid; (4) 2methyl-3-hydroxybutyric acid; (5) urea; (6) phosphoric acid; (7) succinic acid; (8) fumaric acid; (9) glutaric acid; (10) 3-hydroxyadipic lactone (C6:0); (11) adipic acid; (12) 4-hydroxyphenyllacetic acid; (13) octanedioic acid (isomer); (14) octanedioic acid (isomer) and 3hydroxyadipic acid (C6:0); (15) suberic acid; (16) aconitic acid; (17) homovanillic acid; (18) citric acid; (19) hippuric acid (ditrimethylsilyl); (20) hippuric acid (monotrimethylsilyl); (21) 3-hydroxyoctanedioic acid (C8:1); (22) decanoic acid; (23) 3-hydroxysuberic acid (C8:0); (24) sebacic acid; (25) 4-hydroxyphenyllactic acid; (26) pentadecanoic acid (the internal standard); (27) 3-hydroxydecanoic acid (C10:1); (28) 3-hydroxysebacic acid (C10:0); (29) this peak contains 3-hydroxydodecanoic acid (C12:1) and some unknown compounds; (30) 3-hydroxydodecanoic acid (C12:0); (31) 3-hydroxytetradecanoic acid (C14:1); (32) 3-hydroxytetradecanoic acid (C14:0). Identification of all compounds in peaks were confirmed by gas chromatography/mass spectrometry. The analysis is completed in $\mathbf{4 8} \mathrm{min}$.

we analyzed an individual with complete trifunctional protein deficiency and report herein the initial definition of the molecular basis of this disease.

\section{Methods}

Case history. The proband was the product of a term pregnancy complicated by gestational diabetes and hypertension. There was no evidence of maternal AFLP syndrome. Delivery was by emergent caesarean section because of fetal bradycardia. The patient was well for $32 \mathrm{~h}$ but then became lethargic, pale, and hypotonic. After treatment with intravenous glucose, the infant recovered well until the 6th day of life, when, after a change in feeding frequency from every 3 to every $4 \mathrm{~h}$, he was noted to be pale, lethargic, and tachypneic, with hypoglycemia $(25 \mathrm{mg} / \mathrm{dl})$. Symptomatic improvement occurred with intravenous glucose. Echocardiogram demonstrated severe dilated cardiomyopathy (ejection fraction of $40 \%$, normal $>64 \%$ ), for which treatment with dobutamine and digoxin was initiated. Organic acid analysis by capillary gas chromatography/mass spectrometry of a urine specimen obtained immediately after proof of hypoglycemia (Fig. 1A) revealed moderate lactic aciduria

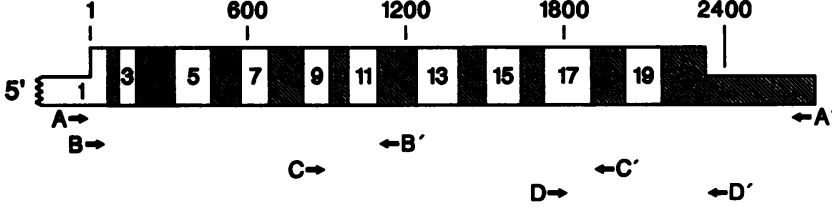

Figure 2. Strategy for RT-PCR amplification of $\alpha$ subunit mRNA. The mRNA of trifunctional protein is depicted to scale with the regions encoded by each exon. ${ }^{2}$ The coding region is the higher vertical box, the untranslated regions are shown at each end of the mRNA in shorter boxes. The 5' end of the mRNA (transcription start site) remains undefined. Sense oligonucleotides for PCR are indicated by the letters $A, B$, $C$, and $D$. Paired antisense oligonucleotides are $A^{\prime}, B^{\prime}, C^{\prime}$, and $D^{\prime}$.

( $558 \mathrm{mmol} / \mathrm{mol}$ creatinine as compared with the range in age-matched controls of 46-348), hypoketotic C6-C10 dicarboxylic aciduria (3hydroxybutyric acid of $47 \mathrm{mmol} / \mathrm{mol}$ creatinine with normal range of $2-45$, adipic acid of 285 with normal of 9-37, octanedioic acid of 30 with no detectable level in normals, suberic acid of 166 with normal. range of 4-20, decanoic acid of 5 with none detected in normals, sebacic acid of 18 with normal of 3-16), and significant C6-C14 3-hydroxydicarboxylic aciduria. Quantitative determination of 3-hydroxydicarboxylic acids was not performed because of unavailability of synthetic standards for calibration. This profile is consistent with, strongly suggestive of, and typical of LCHAD deficiency. The patient was fed a simplified formula (Portagen; Mead Johnson, Evansville, IN) containing medium chain triglycerides with polycose and carnitine supplements. His cardiomyopathy gradually improved, and he remained healthy with normal development until the age of $18 \mathrm{mo}$. After an episode of vomiting, he was hospitalized for observation. In spite of a documented normal blood glucose, the child remained restless, and several hours after admission, he was found dead. Autopsy was normal except for the heart, which had mild left ventricular dilatation, mild myocardial fibrosis, and some acute inflammatory cells consistent with myocarditis. Fatty infiltration of the liver, kidneys, or heart was not present. In postmortem urine (Fig. 1B), lactic acid (164 $\mathrm{mmol} / \mathrm{mol}$ creatinine as compared with normal age-matched controls of $21-38 \mathrm{mmol} / \mathrm{mol}$ creatinine), C6-C10 dicarboxylic acids (adipic acid of $16 \mathrm{mmol} / \mathrm{mol}$ creatinine compared with normal of $1-6 \mathrm{mmol} / \mathrm{mol}$ creatinine, octanedioic acid of $8 \mathrm{mmol} /$ mol creatinine with undetectable levels in normals, suberic acid of 9 $\mathrm{mmol} / \mathrm{mol}$ creatinine with normal of $3-8 \mathrm{mmol} / \mathrm{mol}$ creatinine, and sebacic acid of $5 \mathrm{mmol} / \mathrm{mol}$ creatinine with normal of $3-8 \mathrm{mmol} / \mathrm{mol}$ creatinine), and C6-C14 3-hydroxydicarboxylic acids were mildly elevated.

Cell lines, enzyme assays, DNA, and RNA isolation. Skin fibroblasts were obtained from the affected child. The cells were maintained in minimal essential medium supplemented with $10 \%$ FBS, 2 mM glutamine, and antibiotics. LCHAD, short chain L-3-hydroxyacyl-CoA dehydrogenase and short chain 3-ketoacyl-CoA thiolase activities were measured by the method of Venizelos et al. (16). DNA from the patient's fibroblasts and parents' blood was isolated using an alkaline lysis and proteinase digestion (17). RNA was isolated using the guanidine isothiocyanate method with an added DNase digestion to prevent DNA contamination (18).

Reverse transcriptase PCR of cDNA and cDNA cloning. First-strand cDNA was synthesized using an oligo(dT) primer. The cDNA was then amplified according to the scheme in Fig. 2. PCR was performed with annealing at $55^{\circ} \mathrm{C}$ for $30 \mathrm{~s}$, extension at $72^{\circ} \mathrm{C}$ for $90 \mathrm{~s}$, and denaturation at $94^{\circ} \mathrm{C}$ for $30 \mathrm{~s}$ and 25 cycles. Sense and antisense oligonucleotides indicated by the $\mathrm{A}$ and $\mathrm{A}^{\prime}$ ( Table $\mathrm{I}$ ), respectively, in the figure amplify the entire 2289-bp coding region and 374 bp of untranslated regions. The products of this first reaction were then reamplified with oligonucleotide pairs labeled $\mathrm{B} / \mathrm{B}^{\prime}, \mathrm{C} / \mathrm{C}^{\prime}$, and $\mathrm{D} / \mathrm{D}^{\prime}$ (Fig. 2). All oligonucleotide sequences are given in Table $\mathrm{I}$. Annealing for these reactions was also at $55^{\circ} \mathrm{C}$, but extension at $72^{\circ} \mathrm{C}$ was only for $30 \mathrm{~s}$. This "nesting" of PCRs enhanced the yield of specific products. Most primers were de- 


\begin{tabular}{|c|c|c|}
\hline Sense oligonucleotides & Antisense oligonucleotides & Fragment size \\
\hline A 5 '-gctgtcgactcttcagctcaagAT & $A^{\prime}$ cccgtcaagacgtgggctcgagtttgtgta-5' & $2663 \mathrm{bp}$ \\
\hline B 5'-GGGCGATTGGCATCCTCTAGACCGCTT & B' TTCCCCGATTTCTGATGTGA-5' & $1152 \mathrm{bp}$ \\
\hline C 5'-acagAATTCACAGCGTATGCCATGACTATTCC & C' CCTTCCTAAACTTAAGACTGTACCTATC-5' & $1145 \mathrm{bp}$ \\
\hline D 5'-GCTGGATTCCCTGACCACAAGCTTTGGC & D' ACTCGTCCGGAGTACGGCAGCTGAGTCAG-5' & $582 \mathrm{bp}$ \\
\hline Exon 2 and $35^{\prime}$-ttacaagctttgttatatataaaggtactac & ggacttacctttctacttaagggtataaagg- $5^{\prime}$ & $370 \mathrm{bp}$ \\
\hline
\end{tabular}

Uppercase letters indicate nucleotides from the coding regions of $\alpha$ subunit mRNA. Lowercase letters are from the untranslated regions (oligonucleotides labeled $A$ and $A^{\prime}$ ) or introns (in oligonucleotides $C$ and for exons 2 and 3). Engineered restriction sites for subcloning are underlined.

signed with engineered restriction sites to facilitate cloning into the PGEM3Z plasmid vector for sequence determinations. For the 5 ' partial PCR product, an internal SacI site $100 \mathrm{bp}$ upstream of the primer oligonucleotide was used for digestions and subcloning.

Sequence and single-stranded conformation variance analysis. Sequence analyses used the dideoxy chain termination method (19). To verify that no coding region mutations were present in this patient, we also performed single-stranded conformation variance (SSCV) analysis (19) on DNA amplified from each of the 20 exons of the trifunctional protein $\alpha$ subunit, using appropriate conditions and oligonucleotides $(19) .^{2}$

RNA blot and protein immunoblot analyses. RNA and protein analyses were performed according to standard methods (3).

Population screening for splice site mutations by allele-specific oligonucleotide hybridization. We amplified genomic DNA by PCR from 35 unrelated individuals obtained from Centre pour l'Etude des Polymorphismes Humane pedigrees with intronic oligonucleotides encompassing exons 2 and 3 (Table I). These DNA samples have been studied in detail for human genomic mapping and polymorphisms and are derived from French individuals. The product was then applied to a nylon membrane with a vacuum blotting device and probed with the normal and two mutant oligonucleotides using conditions previously described (3).

\section{Results}

Biochemical characterization of trifunctional protein deficiency. Enzymatic studies of fibroblasts from the proband revealed a deficiency of LCHAD activity $(33.6 \pm 13.6$ [SD] nmol/ min per mg protein, $N=4$; control of $97 \pm 29$ with range of 64-144 nmol/min per $\mathrm{mg}, N=10$ ), with preservation of short chain L-3-hydroxyacyl-CoA dehydrogenase activity (95 \pm 41 $\mathrm{nmol} / \mathrm{min}$ per $\mathrm{mg}$ protein, $N=4$; control of $113 \pm 28$ with range $77-150 \mathrm{nmol} / \mathrm{min}$ per $\mathrm{mg}, N=10$ ) and short chain 3-ketoacylCoA thiolase activity $(17.8 \mathrm{nmol} / \mathrm{min}$ per mg protein, $N=2$; control of $24 \pm 5.4$ with range of $18-31 \mathrm{nmol} / \mathrm{min}$ per $\mathrm{mg}$ protein, $N=10$ ). Immunoblot analysis (data not shown) revealed markedly reduced levels of both $\alpha$ and $\beta$ subunits, as compared with control (Hashimoto, T., personal communication). This result is similar to that reported by Hashimoto and co-workers in complete trifunctional protein deficiency (14). RNA blot analysis of total RNA isolated from the patient's fibroblasts probed with a full-length $\alpha$ subunit cDNA $^{2}$ demonstrated a single band of $\sim 2.6 \mathrm{~kb}$, slightly smaller than $\alpha$ subunit mRNA from a normal control (data not shown).

Characterization of deletions in the $\alpha$ subunit of trifunctional protein. We used reverse transcriptase (RT) - PCR amplification of the $\alpha$-subunit mRNA derived from this patient to define the molecular basis of complete trifunctional protein deficiency. This $2.3-\mathrm{kb}$ mRNA coding region was amplified by nested PCR in three shorter and overlapping fragments using the oligonucleotides depicted in Fig. 2 and listed in Table I. The 5 ' partial fragment derived from the oligonucleotides labeled B/B' (Fig. 2) is 1152 bp long in the normal cDNA (Fig. 3, lane 2).

After amplification of the $5^{\prime}$ fragment with mRNA derived from the proband's fibroblasts, the major product was smaller

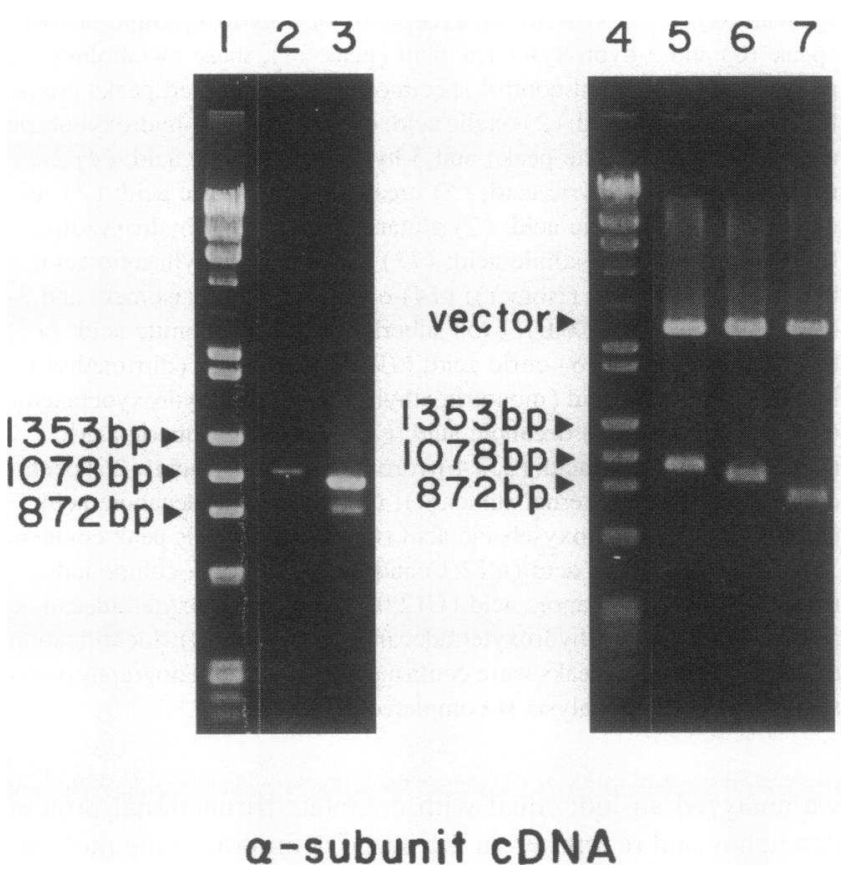

Figure 3. Sizes of 5' partial $\alpha$ subunit PCR products derived from fibroblast RNA from normal and LCHAD deficient cells. This figure is a composite of two different gels, with lanes $1-3$ from one gel and lanes 4-7 from a second gel. Lanes 1 and 4 are marker fragments, with arrows indicating sizes of some fragments. After nested PCR of the 5' fragment with oligonucleotides $A / A^{\prime}$ and $B / B^{\prime}$ shown in Fig. 1, onetenth of the untreated PCR samples from a normal individual (lane 2) and from the LCHAD-deficient proband (lane 3 ) were analyzed on this $1 \%$ agarose gel. After elution of the PCR products from the gel, digestion with restriction enzymes, and placement into plasmid vectors, three different subclones were isolated and digested with restriction enzymes before analysis. The restriction enzyme digestions reduce the size of all the PCR fragments by $117 \mathrm{bp}$. Lane 5 is the partial $5^{\prime}$ product from normal fibroblast mRNA. Lane 6 is one of the five identical clones derived from the patient's 5' fragment of $\alpha$ subunit, which proved to contain the 71-bp exon 3 deletion. Lane 7 is the patient's $5^{\prime}$ partial fragment, which proved to have the 113-bp exon 2-3 deletion. 


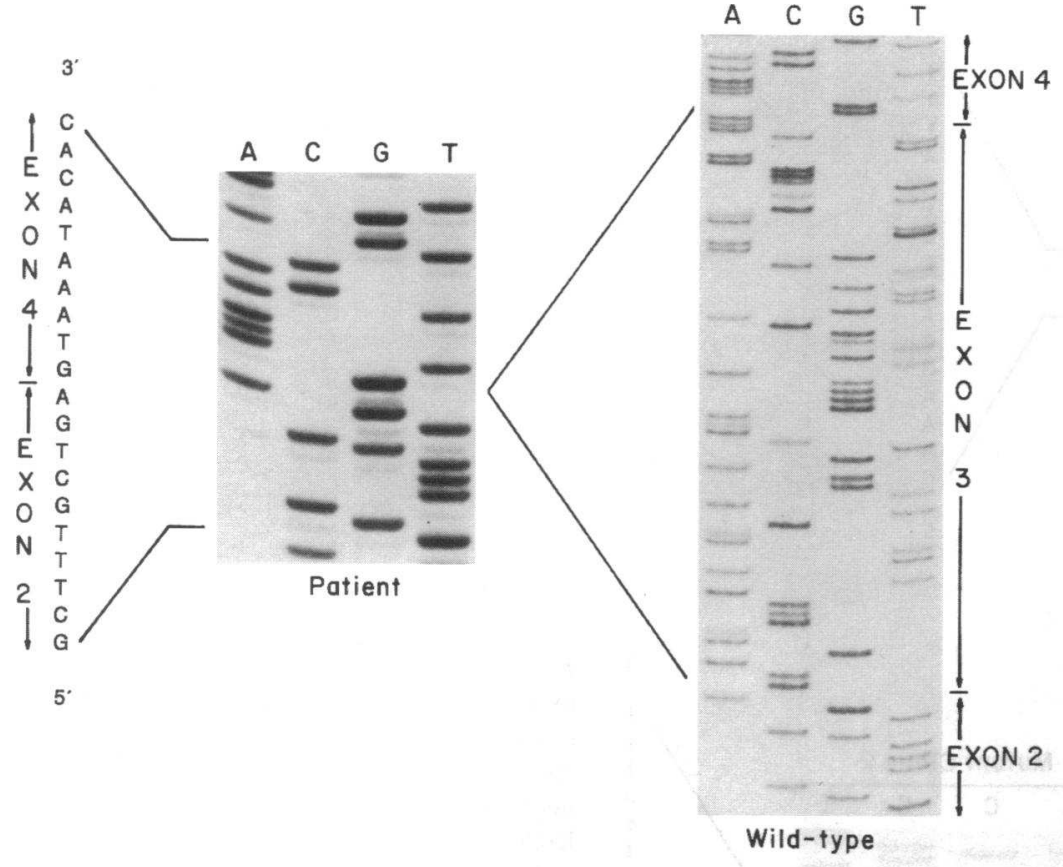

LCHAD CDNA
Figure 4. Nucleotide sequences of the 5' PCR fragments reveal exonic deletions. On the left is a partial sequence of the subcloned 5' PCR fragment derived from fibroblast mRNA of the patient. As indicated to the left of the sequence, comparison with the normal $\alpha$ subunit cDNA and gene reveals deletion of exon 3 . The nucleotide sequence from the comparable region of the subcloned 5' PCR fragment of normal RNA is shown on the right, with sites of splicing of normal exons, as indicated. than expected (Fig. 3, lane 3, upper band), as demonstrated by comparison with the amplified fragment from a normal individual analyzed simultaneously (lane 2 ). No product of the correct size ( $1152 \mathrm{bp}$ ) was present, consistent with the conclusion that no full-length $\alpha$ subunit mRNA is detectable. The difference in mobility of this upper band suggests that 60-80 bp are deleted in the mutant fragment. In addition to the major band, the RTPCR product of this $5^{\prime}$ fragment from the proband contained a second, minor band (Fig. 3, lane 3, lower band). DNA from both bands was digested with $\mathrm{XbaI}$ (in oligonucleotide $\mathrm{B}$ ) and SacI (a naturally occurring site at bp 1068 of the normal mRNA and $80 \mathrm{bp}$ the $5^{\prime}$ direction of oligonucleotide $\mathrm{B}^{\prime}$ ) and placed into plasmid vectors for sequence analyses. The insert size of five patient subclones was identical to (Fig. 3, lane 6) and slightly, but definitely, smaller than the insert derived by PCR from normal mRNA (lane 5). The nucleotide sequences of these five subclones were identical. Sequence comparison with normal human $\alpha$ subunit $\mathrm{cDNA}^{2}$ (20) demonstrated a 71-bp deletion beginning at nucleotide 110 of the mRNA (Fig. 4). This deletion creates a shift in the reading frame of the mRNA. The predicted mutant $\alpha$ subunit mRNA sequence would generate a stop codon (UAA) after 123 bases of coding region sequence. We have recently characterized the intron-exon structure of the human gene encoding the $\alpha$ subunit and defined the splice donor and acceptor sequences surrounding the 20 exons of this gene. ${ }^{2}$ Comparison of the deletion mutation with this trifunctional protein $\alpha$ subunit genomic structure reveals that this 71-bp deletion corresponds precisely to exon 3. A sixth, somewhat smaller subclone insert (Fig. 3, lane 7) was sequenced, and comparison with normal $\alpha$ subunit cDNA revealed a 113-bp deletion. This deletion corresponds precisely to the nucleotides included in exons 2 and 3 of the gene (sequence data not shown). The exon 2-3 deletion also produces a frameshift and creates a premature stop codon after 81 bases of $\alpha$ subunit coding region.
Complete sequence analyses of both strands of subclones (data not shown) derived from the middle and $3^{\prime}$ fragments of the cDNA were also performed. The nucleotide sequences were identical to our normal $\alpha$ subunit cDNA throughout the entire coding region. To discount the possibility of other mutations in this individual's $\alpha$ subunit, we also performed SSCV analysis of DNA amplified from each of the 20 exons of his genomic DNA. No additional conformational differences were detected (data not shown).

These results demonstrate that all of the detectable $\alpha$ subunit mRNA of this patient with trifunctional protein deficiency is abnormal. Based upon the observation that no PCR product or mRNA of appropriate size was found, we hypothesized that the patient had either ( $i$ ) a small deletion(s) of the $\alpha$ subunit gene in the exon 2-3 region, (ii) a donor or acceptor splice site mutation(s) causing exon skipping, or (iii) both. These possibilities were investigated by characterization of the mutant $\alpha$ subunit gene and comparison with the normal gene we have recently isolated. ${ }^{2}$

Definition of two splice site mutations. To identify possible splice site mutations, we amplified genomic DNA encompassing exons 2 and 3 from the proband using oligonucleotides (Table I) derived from sequences in the introns before exon 2 and after exon 3 , but containing engineered restriction sites to allow subcloning. In the normal gene, exon 2 contains 42 bp (base pairs 68-109 of the cDNA), exon 3 contains $71 \mathrm{bp}$ (nucleotides 110-180), and the intervening intron is only 98 bp in length. ${ }^{2}$ The amplified genomic DNA fragment of $360 \mathrm{bp}$ was placed into a plasmid vector, and the complete nucleotide sequences of eight subclones were determined on both strands (Fig. 5). The sequences of the exon 3 -intron 3 junction for the normal (Fig. 5, top) and two different mutant subclones (bottom) are depicted. The two different sequences, derived from the two alleles, were represented equally among the genomic clones. The first allele contains a single base change ( $G$ to 


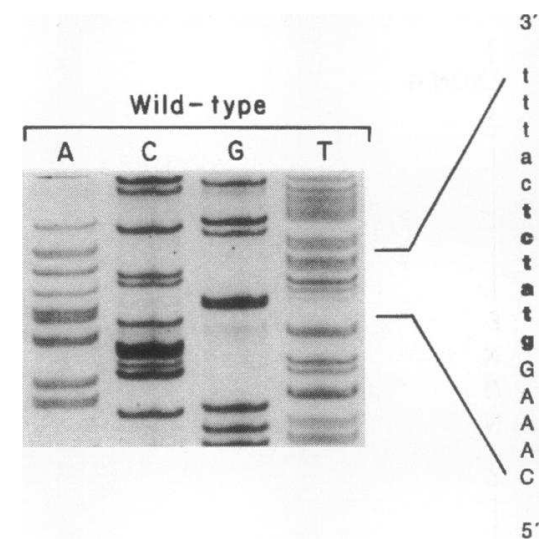

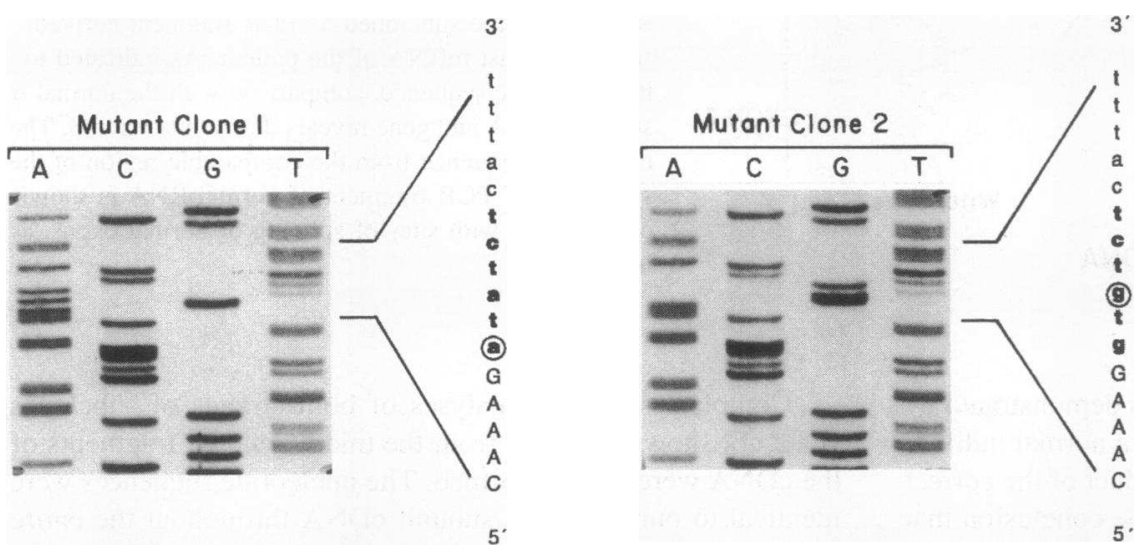

LCHAD Exon 3-Intron 3 Junction
Figure 5. Nucleotide sequences of the exon 3 -intron 3 junction from the normal $\alpha$ subunit gene and from the two mutant alleles of the patient. For all sequences, nucleotides from the exon are in uppercase letters and nucleotides from the intron are in lowercase letters. The consensus donor splice sites are shown in boldface. The normal genomic sequence in this region is shown in the upper half of the figure (designated Wild-type). Sequences from two different subclones derived from the two alleles of the patient are shown in the bottom half of the figure. The single base changes of the two clones that occur in the consensus donor splice site are circled (intron $3-\mathrm{g}+1 \mathrm{a}$ on the left and intron $3-\mathrm{a}+3 \mathrm{~g}$ on the right).
A) at the first intronic base after exon 3 (Fig. 5, bottom left). Similar analysis of parental genomic DNA (data not shown) revealed that the father is heterozygous for this mutant allele and has the normal sequence on his second allele. This change, designated mutation "intron 3-g+1a," occurs within the virtually absolute consensus dinucleotide essential for correct splicing (21). This mutation is therefore consistent with the skipping of exon 3 (missplicing) observed in the proband's mRNA.

The second allele also differs from the normal exon 3 donor splice site sequence. This allele has an A to $\mathrm{G}$ substitution at the third intronic base (Fig. 5, bottom right) and is designated "intron 3-a+3g." Examination of maternal genomic DNA revealed this splice site mutation on one allele, with the normal sequence on her second allele (sequence data not shown). Although this position within the consensus donor splice site sequence (GTAAGT) is more variable than the highly conserved dinucleotide (21), we postulate that this second allelic mutation also causes missplicing. However, we also considered the possibility that this might represent a common polymorphism, which might allow normal splicing of exon 3.

Population screening for splice site mutations. To discount the possibility that the second mutation represented a common polymorphism, we examined DNA from 35 French individuals and the proband. The exon 2-3 regions of genomic DNA were amplified, and the fragments were blotted onto membranes and subjected to hybridization with oligonucleotides containing ei- ther the normal exon 3 splice donor sequence, the intron 3$\mathrm{g}+1 \mathrm{a}$ mutation, or the intron $3-\mathrm{a}+3 \mathrm{~g}$ mutation. Both of the mutant alleles discovered by sequence analysis were detected by this allele-specific oligonucleotide hybridization in the patient. No hybridization with his DNA and the normal oligonucleotide was observed. Genomic DNA fragments from all of the 35 unrelated individuals hybridized to the wild-type oligonucleotide, but not to either of the mutant oligonucleotides (data not shown). These results are consistent with the conclusion that the intron $3-a+3 g$ change in the second allele of this patient is not present in the normal population and thus may cause the observed missplicing, with skipping of exon 3.

\section{Discussion}

Our results demonstrate that this child with fatal trifunctional protein deficiency is a compound heterozygote for two different mutations (intron 3-g+1a and intron $3-a+3 g$ ) in the exon 3 donor splice site. Because neither splice site mutation occurs in the normal population, these base changes do not represent polymorphisms. More importantly, by RT-PCR of the patient's $\alpha$ subunit mRNA and RNA blot analysis, we documented that no detectable normal-sized subunit mRNA is present in his mutant fibroblasts and that his expressed $\alpha$ subunit mRNA is universally deleted for exon 3 . Thus, we conclude that these donor splice site mutations result in incorrect precursor mRNA 
splicing, with deletion of exon 3 or both exons 2 and 3, and production of stable mRNA species containing early premature stop codons.

Naturally occurring mutations of the first intronic base of the splice site donor have been described with deficiency of many gene products, including, for example, lipoprotein lipase (22), phosphofructokinase (23), and ornithine transcarbamylase (24). Mutations at this site may cause use of cryptic splice sites (22) or skipping of the previous exon $(23,24)$, as in the case reported here. Our patient has normal amounts of mRNA by Northern blot, suggesting that in spite of the exonic deletions and premature termination, some mutant mRNA species are stable. In vitro studies of splicing efficiency after systematic creation of mutations at this site demonstrate markedly reduced efficiency of splicing usually secondary to inhibition of the $3^{\prime}$ cleavage and splicing step (25) at the downstream acceptor site. However, some mutations at the +1 site alter the cleavage site. Which of these two abnormalities occurs depends on the entire context at the exon-intron boundaries. Our results support a model (26) in which initial spliceosome assembly requires cooperative interactions ("pairwise recognition of splice sites") at both ends of an exon to form stable complexes and allow exon recognition. This exon scanning mechanism for splice site selection implies that the exon itself is part of the context required for recognition and that the length of the exon $(<300$ bp) is important in determination of splicing.

Disease-causing mutations of the variable bases of the intronic donor splice consensus sequence have been reported previously $(27-30)$. This is somewhat unexpected because the +3 site, for example, is highly variable, with A being the preferred base $(59 \%)$, but with $G$ frequently present $(35 \%)(21)$. Nonetheless, mutations in introns of $\beta$-spectrin at either the +3 or +4 site $(27,28)$ and in the pro- $\alpha 2$ (I) collagen gene at the +5 site (29) produce exon skipping, as in the patient reported here. Moreover, site-specific mutations at the +3 site (30) can abolish normal splicing in vitro. Thus, in this patient, the intron $3-a+3 g$ mutation within the context of this particular splice donor site is sufficient to abolish normal splicing, with resultant exon 3 skipping. We conclude that our patient has misspliced $\alpha$ subunit mRNA, with universal exon 3 skipping, because of two different mutations on two different alleles occurring within the same splice donor site. To our knowledge, this has not previously been described.

The biochemical effect of the aberrant mRNAs produced in this patient by exon skipping is profound. Skipping of exon 3 (or both exons 2 and 3 ) produces mRNA with very early premature termination codons, as documented by RT-PCR of mutant mRNA. Peptides translated from these aberrant mRNAs would contain all or part of the transit or signal peptide required for translocation of the $\alpha$ subunit into mitochondria, but none of the normal 727 amino acids of the mature subunit would be generated. Thus, both the $\mathrm{NH}_{2}$-terminal enoyl-CoA hydratase and the COOH-terminal LCHAD activities would be absent.

The immunoblot result in this patient demonstrates loss of the $\beta$ subunit as well. We propose that absence of all of the mature $\alpha$ subunit would alter the stability of the $\beta$ subunit, perhaps by interfering with appropriate localization to the membrane in mitochondria. Alternatively, because the absence of any normal $\alpha$ subunit precludes heterooctamer formation and because the free $\beta$ subunit undoubtedly has a conformation different from that of the normally multimerized $\beta$ subunit, it is possible that free $\beta$ subunit would be highly susceptible to proteolysis. Hashimoto and co-workers have studied the biosynthesis, processing, heteromeric complex formation, and stability of the $\alpha$ and $\beta$ subunits of this patient and two others with complete loss of trifunctional protein antigen (Hashimoto, T., personal communication). Their results demonstrate that the mutations described here and three different missense mutations in the $\beta$ subunit alleles of two other patients interfere with the formation and stability of the trifunctional protein complex and result in rapid degradation of both subunits in patients' fibroblasts. Similar effects of mutant subunits on the stability of normal subunits in heteromeric complexes, including electron transfer flavoprotein, have been noted (31). Thus, the presence of early premature stop codons in the $\alpha$ subunit of this patient alters the interaction with the thiolase-containing $\beta$ subunit, reducing the formation or stability of the trifunctional protein complex and thus resulting in loss of all three enzymatic activities.

The patient reported here presented with neonatal hypoglycemia and cardiomyopathy. We and others $(3,32)$ have documented that other $\beta$-oxidation defects, especially MCAD deficiency, may also cause neonatal symptoms or death associated with hypoglycemia and fatty infiltration of the heart and liver. Our LCHAD-deficient patient later died suddenly and unexpectedly, a phenotype typical both of isolated LCHAD deficiency and of other defects in $\beta$-oxidation. The similarity of symptoms of our patient and those previously reported with isolated LCHAD deficiency suggests that the LCHAD activity of trifunctional protein is most critical to the normal function of those tissues with high energy demands. Perhaps this is because more redundancy of the other two enzyme activities (enoylCoA hydratase and $\beta$-ketoacyl-CoA thiolase) exists, secondary to multiple gene products encoding various enzymes with these activities (8). An important difference between this case and many autopsy studies of toddlers dying from $\beta$-oxidation defects is that no significant microvesicular fatty infiltration of any organ was detected. The mild myocardial fibrosis and superimposed myocarditis suggest that an arrhythmia may have been responsible for death, although this is speculative.

In summary, our results demonstrate that two different mutations in the exon 3 donor splice site of the trifunctional protein $\alpha$ subunit gene cause exon skipping and creation of very early premature stop codons in the misspliced, but stable, mature mRNA. The resultant absence of $\alpha$ subunit allows rapid degradation of the normal $\beta$ subunit and creates a deficiency of all three trifunctional protein enzyme activities. This is the initial delineation of the molecular basis of LCHAD and trifunctional protein deficiency.

\section{Acknowledgments}

We thank Daniel Kelly for critical review of this manuscript. Gypsyamber Berg-Cross, Heidi Rayala, and Michael Weinberger completed some of the experiments reported in this paper.

This study was supported by a grant from the National Institutes of Health (AM20407). J. C. Brackett was supported by a National Institutes of Health institutional training grant (T32-HL07081-20).

\section{References}

1. Hale, D. E., and M. J. Bennett. 1992. Fatty acid oxidation disorders: a new class of metabolic diseases. J. Pediatr. 121:1-11.

2. Tanaka, K., I. Yokota, P. M. Coates, A. W. Strauss, D. P. Kelly, Z. Zhang, N. Gregersen, B. S. Andresen, Y. Matsubara, D. Curtis, and Y. T. Chen. 1992. 
Mutations in the medium chain acyl-CoA dehydrogenase gene. Hum. Mutation 1:271-279.

3. Brackett, J. C., H. F. Sims, R. D. Steiner, M. Nunge, E. M. Zimmerman, B. deMartinville, P. Rinaldo, R. Slaugh, and A. W. Strauss. 1994. A novel mutation in medium chain acyl-CoA dehydrogenase causes sudden neonatal death. J. Clin. Invest. 94:1477-1483.

4. Riudor, E., A. Ribes, M. Borowat, C. Sabado, C. Dominquez, and A. Ballabriga. 1986. A new case of familiar C6-C14 dicarboxylic aciduria with favorable evolution. J. Inher. Metab. Dis. 11:183-185.

5. Pollitt, R. J., H. Losty, and A. Westwood. 1987. 3-OH-dicarboxylic aciduria. J. Inher. Metab. Dis. 10:266-269.

6. Kelley, R. I., and D. H. Morton. 1988. 3-OH-octanoic aciduria: identification of a new organic acid in the urine of a patient with non-ketotic hypoglycemia Clin. Chim. Acta. 175:19-26.

7. Wanders, R. J. A., L. IJlst, M. Duran, C. Jakobs, J. B. C. deKlerk, H. Przyrembel, F. Rocchiccioli, and P. Aubourg. 1991. Long chain 3-hydroxy-acylCoA dehydrogenase deficiency: different clinical expression in three unrelated patients. J. Inher. Metab. Dis. 14:325-328.

8. Jackson, S., R. S. Kler, K. Bartlett, H. Briggs, L. A. Bindoff, M. Pourfarzam, D. Gardner-Medwin, and D. M. Turnbull. 1992. Combined defect of mitochondria fatty acid oxidation. J. Clin. Invest. 90:1219-1225.

9. Wilcken, B., K.-C. Leung, J. Hammond, R. Kamath, and J. V. Leonard. 1993. Pregnancy and fetal long chain 3-hydroxy-acyl-CoA dehydrogenase deficiency. Lancet. 341:407-408

10. Treem, W. R., P. Rinaldo, D. E. Hale, C. A. Stanley, D. S. Millington J. S. Hyams, S. Jackson, and D. M. Turnbull. 1994. Acute fatty liver of pregnancy and long chain 3-hydroxy-acyl-CoA dehydrogenase deficiency. Hepatology. 19:339-345.

11. Carpenter, K., R. J. Pollitt, and B. Middleton. 1992. Human long chain 3-hydroxy-acyl-CoA dehydrogenase is a multifunctional membrane-bound $\beta$-oxidation enzyme of mitochondria. Biochem. Biophys. Res. Commun. 183:443-448.

12. Uchida, Y., K. Izai, T. Orii, and T. Hashimoto. 1992. Novel fatty acid $\beta$ oxidation enzymes in rat liver mitochondria. J. Biol. Chem. 267:1034-1041.

13. Kamijo, T., T. Aoyama, J. Miyazaki, and T. Hashimoto. 1993. Molecula cloning of the cDNAs for the subunits of rat mitochondrial fatty acid $\beta$-oxidation multienzyme complex. J. Biol. Chem. 268:26452-26460.

14. Kamijo, T., R. J. A. Wanders, J.-M. Saudubray, T. Aoyama, A. Komiyama and T. Hashimoto. 1994. Mitochondrial trifunctional protein deficiency. J. Clin. Invest. 93:1740-1747.

15. Jackson, S., K. Bartlett, J. Land, E. R. Moxon, R. J. Pollitt, J. V. Leonard, and D. M. Turnbull. 1991. Long chain 3-hydroxy-acyl-CoA dehydrogenase deficiency. Pediatr. Res. 29:406-411.

16. Venizelos, N., L. IJlst, R. J. A. Wanders, and L. Hagenfeldt. 1994. $\beta$ Oxidation enzymes in fibroblasts from patients with 3-hydroxydicarboxylic aciduria. Pediatr. Res. 36:111-114.

17. Ausubel, F. M., R. Brent, R. E. Kingston, D. D. Moore, J. G. Seidman,
J. A. Smith, and K. Struhl. 1994. Current Protocols in Molecular Biology. John Wiley \& Sons, Inc. New York. 1.7.1-1.7.15.

18. Chomczynski, P., and N. Sacchi. 1987. Single-step method of RNA isolation by acid guanidinium thiocyanate-phenol-chloroform extraction. Anal. Biochem. 162:156-159.

19. Orita, M., H. Iwahana, H. Kanazawa, K. Hayashi, and T. Sekiya. 1989. Detection of polymorphisms of human DNA by gel electrophoresis as singlestrand conformation polymorphisms. Proc. Natl. Acad. Sci. USA. 86:2766-2770.

20. Kamijo, T., Aoyama, A. Komiyama, and T. Hashimoto. 1994. Structural analysis of cDNAs for subunits of human mitochondrial fatty acid $\beta$-oxidation trifunctional protein. Biochem. Biophys. Res. Commun. 199:818-825.

21. Senapathy, P., M. B. Shapiro, and N. L. Harris. 1990. Splice junctions, branch point sites, and exons. Methods Enzymol. 183:252-270.

22. Gotoda, T., N. Yamada, T. Murase, T. Inaba, S. Ishibashi, H. Shiman, S. Koga, Y. Yazaki, T. Furuichi, and F. Takaku. 1991. Occurrence of multiple aberrantly spliced mRNAs upon a donor site mutation that causes familial lipoprotein lipase deficiency. J. Biol. Chem. 266:24757-24762.

23. Raben, N., J. Sherman, F. Miller, H. Mena, and P. Plotz. 1993. A 5 ' splice junction mutation leading to exon deletion in a Ashkenazic Jewish family with phosphofructokinase deficiency. J. Biol. Chem. 268:4963-4967.

24. Carstens, R. P., W. A. Fenton, and L. R. Rosenberg. 1991. Identification of RNA splicing errors resulting in human ornithine transcarbamylase deficiency. Am. J. Hum. Genet. 48:1105-1114.

25. Sharp, P. A. 1994. Split genes and RNA splicing. Cell. 77:805-815.

26. Niwa, M., C. C. MacDonald, and S. M. Berget. 1992. Are vertebrate exons scanned during splice-site selection? Nature (Lond.). 360:277-280.

27. Garbarz, M., W. T. Tse, P. G. Gallagher, C. Picat, M.-C. Lecomte, F. Galibert, D. Dhermy, and B. G. Forget. 1991. Spectrin Rouen, a novel shortened $\beta$-chain variant in a kindred with hereditary elliptocytosis. J. Clin. Invest. 88:7681 .

28. Gallagher, P. G., W. T. Tse, F. Costa, A. Scarpa, P. Boivin, J. Delaunay, and B. G. Forget. 1991. A splice site mutation of the $\beta$-spectrin gene causing exon skipping in hereditary elliptocytosis associated with a truncated $\beta$-spectrin chain. J. Biol. Chem. 266:15154-15159.

29. Ganguly, A., C. T. Baldwin, D. Strobel, D. Conway, W. Horton, and D. J. Prockop. 1991. Heterozygous mutation of the $\mathrm{G}^{+5}$ position of intron 33 of the pro- $\alpha 2$ (I) gene that causes aberrant RNA splicing and lethal osteogenesis imperfecta. J. Biol. Chem. 266:12035-12040.

30. Montell, C., and A. J. Berk. 1984. Elimination of mRNA splicing by a point mutation outside the conserved GU at $5^{\prime}$ splice sites. Nucleic Acids Res. 12:3821-3827.

31. Frerman, F. E., and S. I. Goodman. 1985. Deficiency of electron transfer flavoprotein or electron transfer flavoprotein: ubiquinone octoreductase in glutaric aciduria type II fibroblasts. Proc. Natl. Acad. Sci. USA. 82:4517-4520.

32. Wilcken, B., K. H. Carpenter, and J. Hammond. 1993. Neonatal symptoms in medium chain acyl-CoA dehydrogenase deficiency. Arch. Dis. Child. 69:292294. 